\title{
Effect of Cation Point Defects in Doped Ceria Materials on Surface Oxygen Vacancies and Exchange Reactions
}

\author{
Mai $\operatorname{Tan}^{1}$ and Peter Crozier ${ }^{2}$ \\ ${ }^{1}$ Arizona State University, CHANDLER, Arizona, United States, ${ }^{2}$ Arizona State University, Tempe, \\ Arizona, United States
}

Ceria and ceria-based materials are well known for their unique ability of reversibly exchanging oxygen with the surrounding environment while changing oxidation state and maintaining structural integrity, allowing it to be widely used in many catalytic, energy and sensing technologies. Because of the highlevel tolerance to oxygen vacancies in ceria-based material, they have been commonly doped with aliovalent dopants, such as $\mathrm{Pr}^{3+}, \mathrm{Gd}^{3+}$ and $\mathrm{Ca}^{2+}$, to increase extrinsic oxygen vacancies resulting in enhanced ionic and electronic conductivity resulting in [1]. The degree of mixed ionic-electronic conductivity is associated with oxygen partial pressure, temperature, and the presence of oxygen vacancies created by cation dopants. For applications that require high ionic conductivity, such as solid oxide fuel cells, thermally activated oxygen ions migrate via vacancy hopping and exchange with gaseous oxygen. However, the association between different cation defects and oxygen vacancies is not well understood. It is important to develop atomic level spectroscopy technique to correlate the cation point defect location and oxygen exchange reaction at the surface.

5\%, 15\% of Pr-doped ceria (PDC), Gd-doped ceria (GDC) and undoped ceria were synthesized using solution-based hydrothermal method[2]. Since oxygen exchange involves vacancies and electrons, Gd is selected for its strong ionic conduction ability and Pr is selected for its mixed ionic and electronic conduction. Mixtures of nitrates and hydroxides were heated under autogenous pressure for $10 \mathrm{hr}$ in autoclave. The resulting nanoparticles were characterized before and after calcination at $370{ }^{\circ} \mathrm{C}$ for $2 \mathrm{hr}$ in JEOL 2010F microscope and PANalytical X-ray diffractometer. Figure 1 showed the TEM images of after calcination GDC, PDC, ceria nanoparticle in [110] projection. Comparing all the surfaces for those three materials, sawtooth-like (111) nano-facet were dominated on the (110) surfaces. Both experimental studies and theoretical calculations have determined that (111) surfaces have the lower surface energy than (110) surfaces [2,3]. The rearrangement of (110) surface into (111) nano-facet can reduce the high surface energy. Figure 2 showed the electron energy-loss spectrum (EELS) of 15\% Gd-doped ceria acquired from JEOL ARM200F microscope, which Ce M4,5 and Gd M4,5 edges were identified.

Our goal is to examine the oxygen exchange properties with various dopants under different temperatures. Since the diffusion kinetics become much more rapid at high temperature, it is feasible to characterize the oxygen exchange rate using isotopic exchange methods with mass spectrometry in future experiments. Furthermore, grain boundary dopant segregation has been shown to occur ceria-doped material at high temperature [5]. To more easily investigate the surface relationship between cation dopants and oxygen vacancies, we will promote cation surface segregation by exposing the materials to different heat treatments. We will use identical location STEM EELS to track the difference in the chemical composition of the surface before and after heat treatments. Phase contrast imaging techniques will be used to locate oxygen vacancies associated with surface cation dopants. 

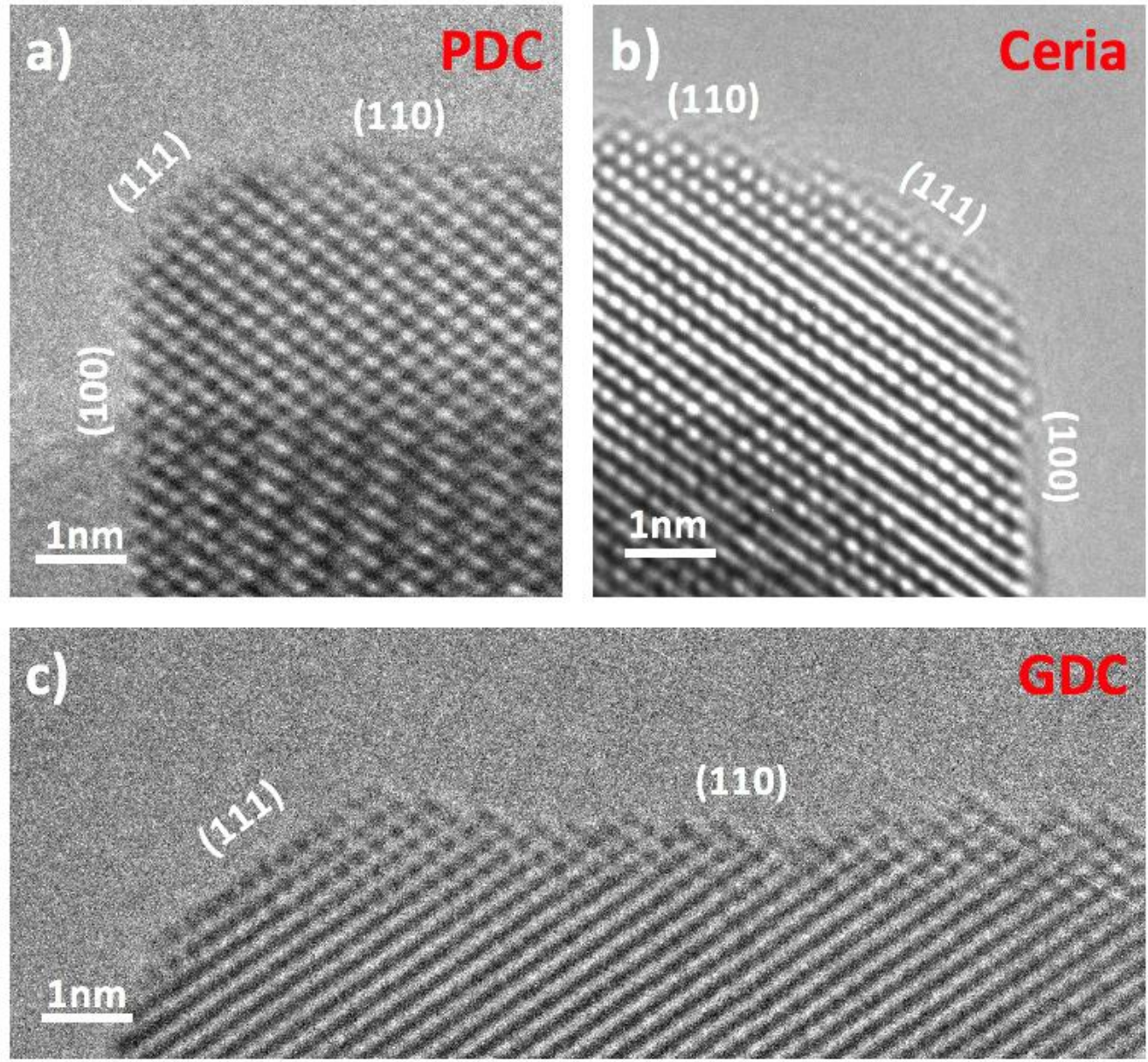

Figure 1. Figure 1: TEM images of after calcination a) 15\% Pr-doped ceria b) ceria c) $15 \%$ Gd-doped ceria nanoparticle in [110] projection. Some of the crystal surfaces of the particles are labeled. 


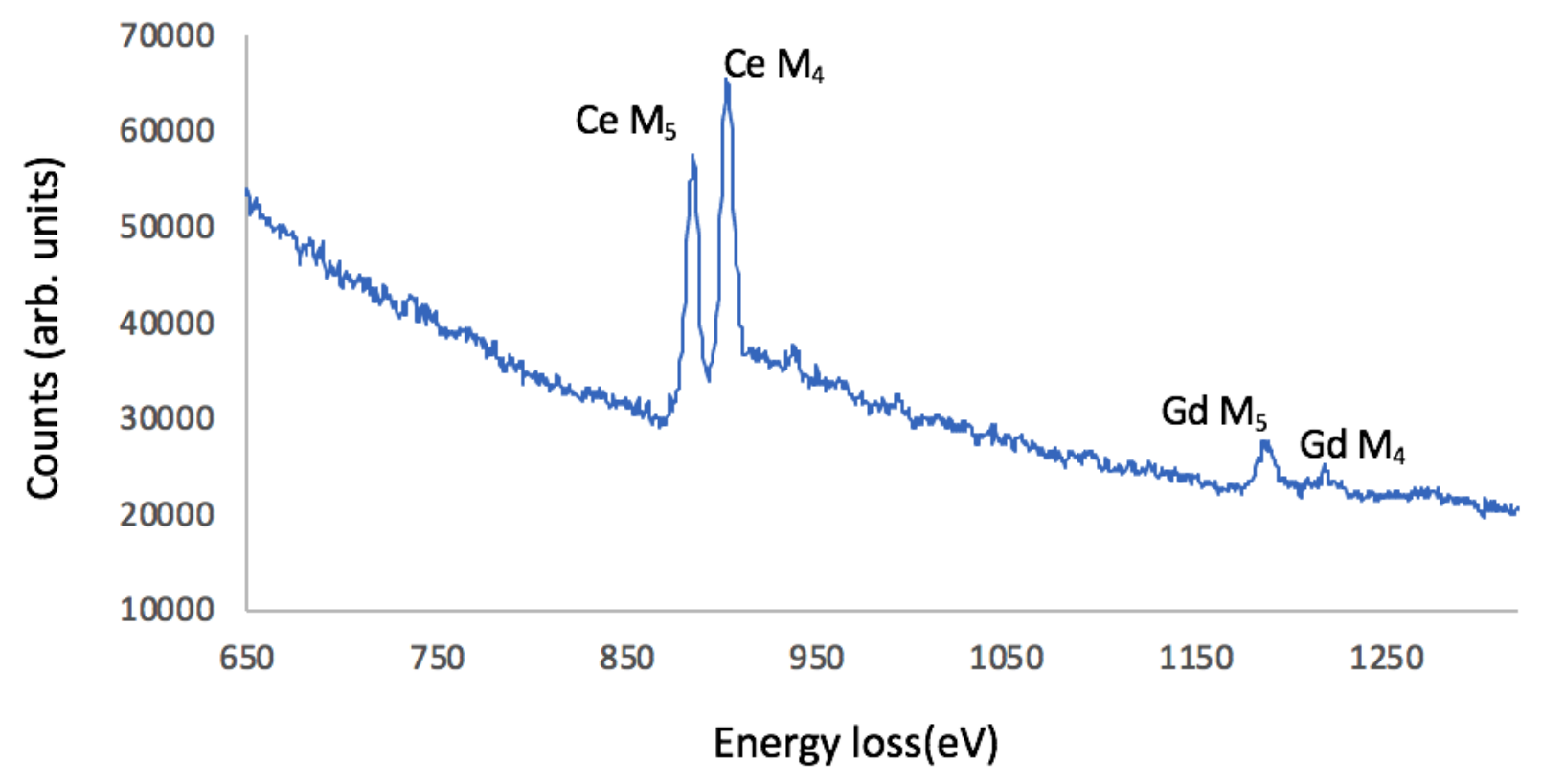

Figure 2. Figure 2: EELS spectrum of $15 \%$ Gd-doped ceria at the surface. Ce M4,5 and Gd M4,5 edges are identified.

\section{Reference:}

[1] Montini, T., Chemical Reviews, 116(2016), 5987-6041

[2] Mai,H., Journal of Physical Chemistry B, 109(2005), 24380-24385

[3] Paier, J., Chemical Reviews, 113(2013), 3949-3985

[4] Baudin, M., Surface science, 468(2000), 51-61

[5] Bowman, W., Solid State Ionics, 272 (2015): 9-17.

[5] The authors acknowledge funding from NSF DMR 1840841, and the use of facilities of Eyring Materials Center at Arizona State University 